\title{
Electrochemical blackening of yttria-stabilized zirconia - morphological instability of the moving reaction front
}

\author{
J. Janek*, C. Korte
}

\begin{abstract}
Electrochemical reduction was performed on both polycrystals of calcia-stabilized zirconia (CSY) and single crystals of yttria-stabilized zirconia (YSZ) at $500^{\circ} \mathrm{C}$. A glass-encapsulation was used to prevent access of molecular oxygen from the gas phase and to realize a virtually two-dimensional geometry for the reacting crystal. The resulting blackening process which occurs by the advancement of a morphologically unstable reaction front was observed in-situ in a heating-stage by the use of an optical microscope.
\end{abstract}

Keywords: Mixed conductors; Electrocoloration; Solid electrolytes; Zirconia

\section{Introduction}

Coloration (blackening) of cubic stabilized zirconia caused by strong chemical or electrochemical reduction is a well known phenomenon. Since its first observation in the course of the development of MHD-devices (magneto-hydrodynamic devices [1]) numerous investigations were performed on blackened zirconia (mostly at room temperature), particularly to clarify the nature of the blackened material [1-23]. Thus optical spectroscopy [3-6], EPR measurements [6-18], conductivity measurements $[1,6,19,20]$, electron microscopy [21], X-ray studies [7], dielectric measurements [22] and measurements of elastic properties $[23,24]$ were performed by different groups. Blackening is also observed during laser treatment of zirconia ceramics

\footnotetext{
*Corresponding author. Tel.: +49-511-762-5298; fax: +49511-762-4009; e-mail: janek@mbox.pci.uni-hannover.de
}

[25] and coulometric titrations with zirconia as a solid electrolyte [26], indicating strongly reducing conditions. Despite these studies the chemical nature of blackened zirconia is still in discussion and attracts further interest [27].

Mostly, during electrochemical reduction a zirconia specimen is surrounded by an inert gas atmosphere with unknown oxygen activity while a sufficiently high electric voltage is applied via platinum electrodes to the crystal. The typical result of this procedure is a tongue-shaped blackened region in the center of the specimen since the surfaces of the specimen can still act as oxygen sources. Essentially, the activity of oxygen in the reduced material is controlled kinetically rather than thermodynamically. Since different geometric boundary conditions have been realized by different authors, the results of reduction experiments vary considerably. Furthermore, as a consequence of the simple design of the usual electrochemical polarisation cells, no reliable 
information on both the properties of reduced zirconia (as functions of the thermodynamic variables) and the electrochemical reduction process itself has been obtained. It is the major purpose of this paper to present results of an experimental study with well-defined boundary conditions for the electrochemical reduction, and thus, under reproducible thermodynamic conditions. We avoid oxygen exchange by the use of a virtually oxygen-impermeable glass cover encapsulating the zirconia specimen.

The electrochemical reduction of zirconia by application of an electric field in an inert atmosphere with a non-zero oxygen activity corresponds to a partially blocking electrochemical polarization cell (Wagner-Hebb cell, e.g. see [28]). The free surfaces of the mixed conductor (CSZ, YSZ) can act as additional sources or sinks for oxygen and reduce the polarizing effect of the applied electric field (cf. [29]). By encapsulating our cells in glass we obtain ideal polarisation cells which can formally be described by relations according to Wagner and Hebb [30,31].

The electrochemical blackening of zirconia provides an example of internal solid state reactions driven by an electric field, which have been introduced by Schmalzried [32,33]. In the course of such internal reaction, point defects (charged oxygen vacancies and electrons in the present case) migrate as educts from the boundaries of the crystalline reaction medium to the reaction zone and form the colorating product. It is the further purpose of this paper to demonstrate an internal electrochemical reaction and its morphological development. The encapsulation of thin zirconia specimens in a (transparent) glass cover allows to study the reaction in a quasi-two dimensional geometry and to observe the moving and morphological unstable reaction front directly with an optical microscope in situ.

Electrochemical reduction has been already studied in a number of other oxides, mainly due to technological interest. Extensive experimental and numerical studies on the electrochemical degradation of earth alkali titanates have been reported by Waser et al. [34]. Due to a slow surface reaction at low temperatures the advancing coloration front could be observed without the application of an additional impermeable surface cover. The direct observation of internal precipitation of bromine in polarized silver bromide crystal has been reported by Stilkenböhmer and Schmalzried [35,36] together with a formal analysis of the underlying kinetics.

\section{Experimental}

\subsection{Specimens}

Polycrystalline $\mathrm{ZrO}_{2}(10 \mathrm{~mol} \% \mathrm{CaO})$ was delivered by Friedrichsfeld (Mannheim, Germany), and single crystals of $\mathrm{ZrO}_{2}\left(12 \mathrm{~mol} . \% \quad \mathrm{Y}_{2} \mathrm{O}_{3}\right)$ were purchased from Kristallhandel Kelpin (Leimen, Germany). Both single and polycrystals were cut into thin rectangular pieces $\left(\sim 0.5 \times 5 \times 20 \mathrm{~mm}^{3}\right)$ with a diamond saw. The surfaces of the single crystals were polished with diamond paste $(1 \mu \mathrm{m})$ to achieve good transparency for optical transmission microscopy. A small piece of platinum foil (cathode) was sintered to one small edge of the specimen with the help of platinum paste. Platinum wire was used for the electrical contacts (see Fig. 1).

Each crystal was placed separately in a small platinum crucible and covered with coarse grained pieces of glass (optical alkali-lead-silicate glass F2, SCHOTT Glaswerke, Mainz/Germany). The melting process was performed at $800^{\circ} \mathrm{C}$ for $24 \mathrm{~h}$ to avoid the later enclosure of bubbles. Afterwards the temperature was slowly decreased (within $6 \mathrm{~h}$ ) to $500^{\circ} \mathrm{C}$ and again held constant for $24 \mathrm{~h}$ in order to reduce mechanical stress which always develops due to slightly different thermal expansion coefficients of both crystals and glass.

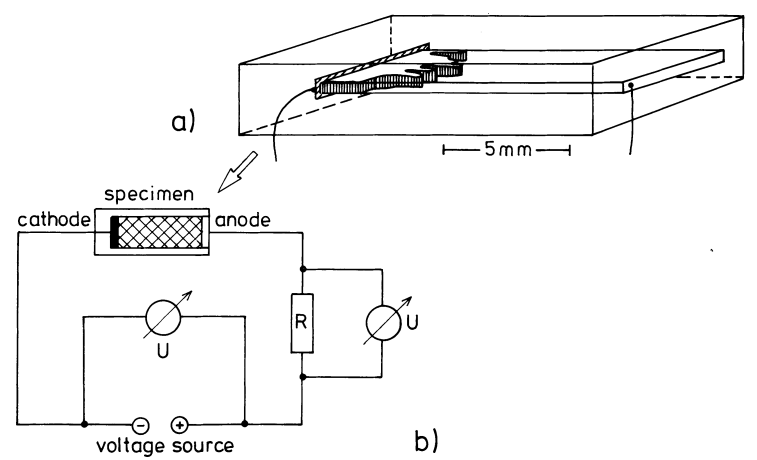

Fig. 1. (a) Electric circuit of the polarization cell, (b) encapsulated specimen. 
After cooling to room temperature the glass bodies were cut to their final shape and polished to achieve good optical quality of the surfaces. The encapsulated single crystalline specimens were rather fragile, and they broke always during the sawing process due to internal mechanical stress of the glass body. Polycrystalline specimens were less sensitive against mechanical stress. No polycrystalline sample was broken during polishing or sawing. Finally, a platinum gauze and a platinum wire were fixed with silver paste at the open end of the cell to produce the anode which is reversible for exchange of oxygen.

\subsection{Experimental set-up}

The electric circuit and the cell design is depicted schematically in Fig. 1. A heating-stage allowed the observation in transmission by an optical microscope. The temperature was limited to $500^{\circ} \mathrm{C}$ mainly by the softening of the glass encapsulation at higher temperatures. The polarization cells were connected to a DC-voltage source as is shown in Fig. 1. A voltmeter was used for the measurement of the applied voltage and a resistance in series with the specimen was used as a shunt for the measurement of the electric current.

\subsection{Experiments}

Several encapsulated specimens were reduced at different applied voltages $\left(T=500^{\circ} \mathrm{C}\right)$. The blocking electrode within the glass body was polarized cathodically. The moving blackening front was documented by photography. The electric current during reduction was measured, and frequently the EMF of partially reduced specimens was measured under open circuit conditions.

\section{Results}

Typical results for the final state of electrochemically reduced polycrystals are shown in Fig. 2. All blackening fronts became morphologically unstable but show different shapes depending on the applied voltage. Cells with embedded single crystals often broke, and most results are obtained from polycrystals. No evidence is given for the participation of the glass cover itself in the electrochemical reaction. Also no evidence is given for a disturbance by diffusion of oxygen through the glass cover.

\subsection{Polycrystalline specimen}

A polarisation with an applied voltage of $10 \mathrm{~V}$ led to a very slow blackening of zirconia. The front of the blackened zone moved almost uniformly with a very slight curvature. After $24 \mathrm{~h}$ the blackened zone extended to $\sim 1 \mathrm{~mm}$ from the cathode. Thus, electrochemical reduction took place but proceeded very slowly and with an almost stable front.

Polarisation at an applied voltage of $100 \mathrm{~V}$ led to much higher reduction rates. The morphology of the reaction front became unstable from the very beginning on and shows characteristic finger-like structures, see Fig. 3a-f. The reduction was completed within several hours, i.e. within this time the blackening front had reached the anode.

Polarisation at an applied voltage of $500 \mathrm{~V}$ led to a very fast reduction process. Within $1 \mathrm{~h}$ the unstable blackening front reached the anode and led to a current breakthrough. The dendrite-like structure which developed during the reduction is again different from structures at lower polarisation voltages.

\subsection{Single crystals}

Polarisation at an applied voltage of $100 \mathrm{~V}$ led to finger-like structures (see Fig. 4a-f) which are very similar to the ones observed in the ceramic specimen. But compared to the slow reduction of the polycrystalline specimen at $100 \mathrm{~V}$, the blackening front in the single crystal moves much faster. The current breakthrough by a contact between the blackened zone and the platinum anode was reached within $10 \mathrm{~min}$, i.e. the reduction proceeds 40 times faster than in the polycrystalline specimen.

Polarisation at an applied voltage of $500 \mathrm{~V}$ again led to a faster reduction and to the development of a finer structure of the reaction front. The current breakthrough was observed within 2 min which is again much faster than in the polycrystalline material.

All blackened regions show sharp boundaries with the unreduced white (transparent) zirconia. Frequent- 


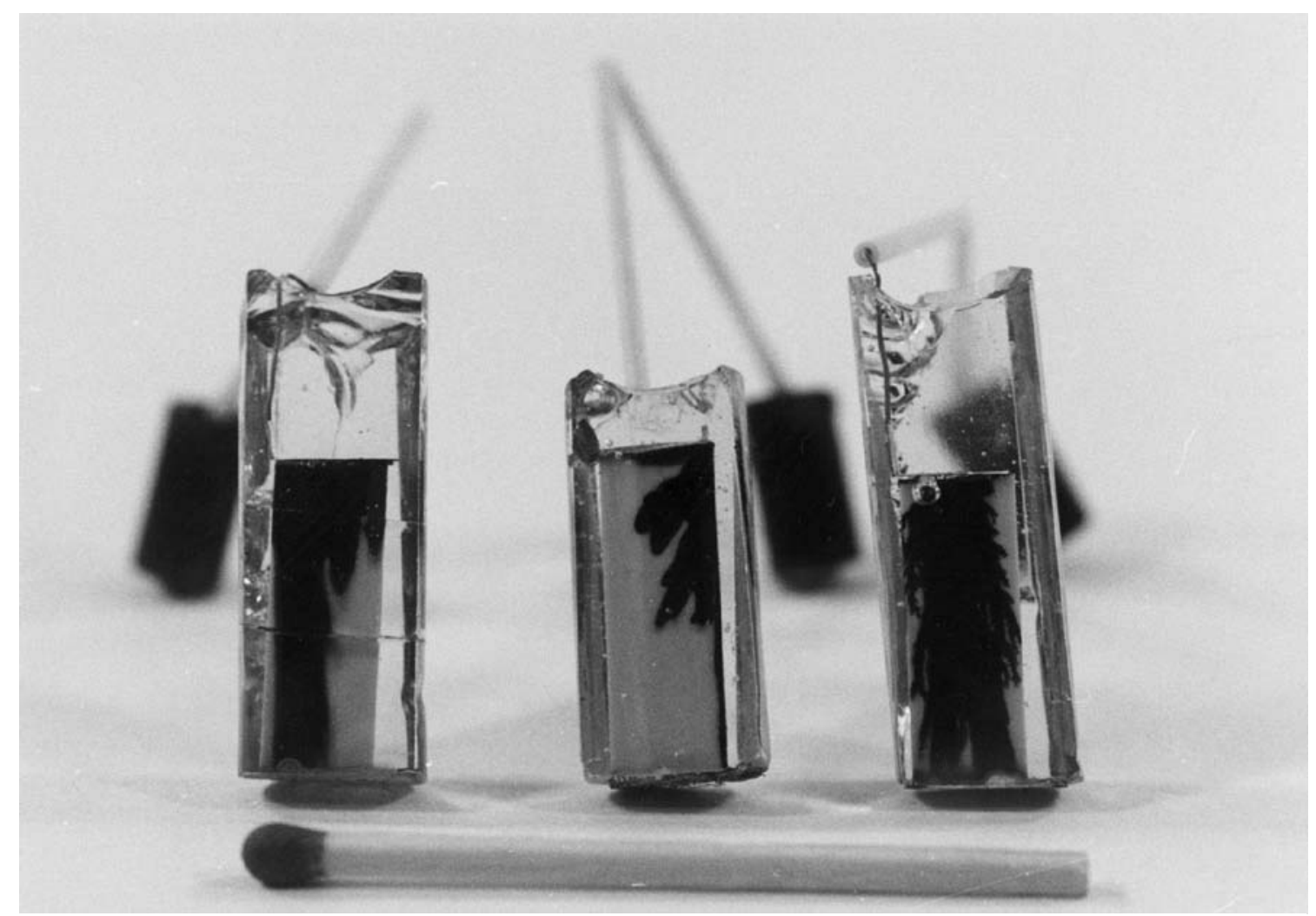

Fig. 2. Three cells after electrochemical reduction at different voltages. Experimental conditions: $T=500^{\circ} \mathrm{C}$, (left and middle cell) $U=100$ $\mathrm{V}$, (right cell) $U=500 \mathrm{~V}$.

ly, partially reduced specimens were disconnected from the voltage source and the remaining open circuit EMF of the cell was measured by means of a voltmeter with high input impedance. Immediately after disconnection of the voltage source the EMF values were as high as 2.0-2.5 V but decreased rapidly to values between $1.0-1.5 \mathrm{~V}$.

\subsection{Electrochemical measurements}

Depending on the applied voltage the electric current shows different behaviour. At small voltages below $2 \mathrm{~V}$ no blackening takes place, and the electronic current decreases until it reaches a stationary value. At higher voltages electrochemical blackening takes place, and the advancing reduction zone leads to an increasing current. This current involves both the electronic partial current of the polarized crystal and the ionic partial current which is related directly to the reduction rate. In the present study we made no attempt to separate these contributions. However, it is possible to detect the amount of oxygen which leaves the crystal by suitable techniques as was done by Levy et al. [20].

\section{Formal description}

In pure and stabilized zirconia, extrinsic defects in the oxygen sublattice can be created by the reaction with molecular oxygen,

$\frac{1}{2} \mathrm{O}_{2}+\mathrm{V}_{\mathrm{O}}^{\times} \rightleftharpoons \mathrm{O}_{\mathrm{O}}^{\times}$

i.e. by the loss of molecular oxygen (reduction) oxygen vacancies are created. These oxygen vacancies increase the diffusion coefficient of oxygen ions and therefore also the ionic conductivity of pure 


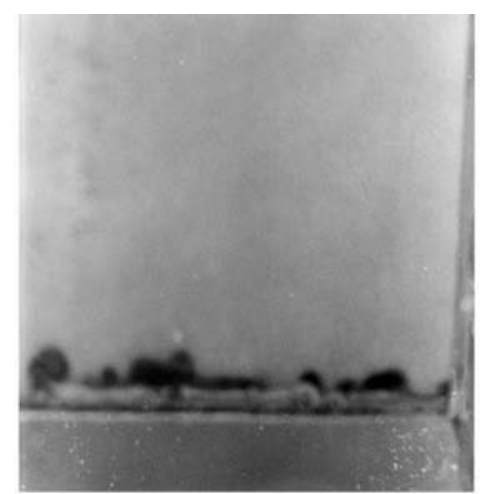

a)

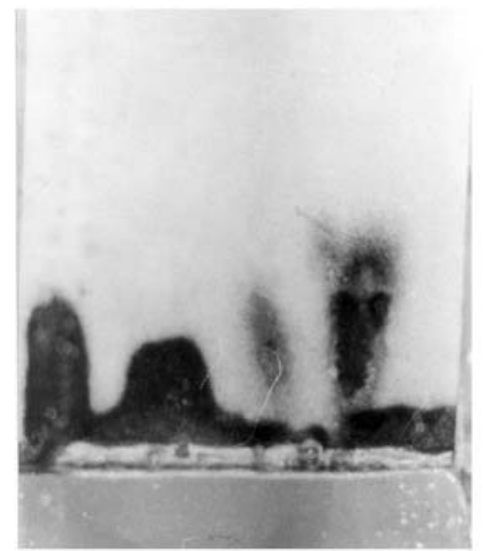

d)

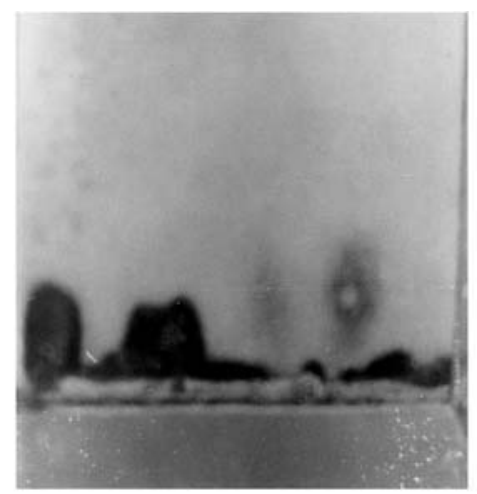

b)

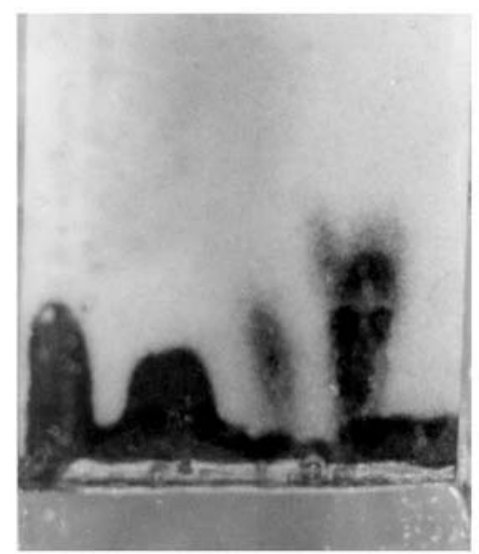

e)

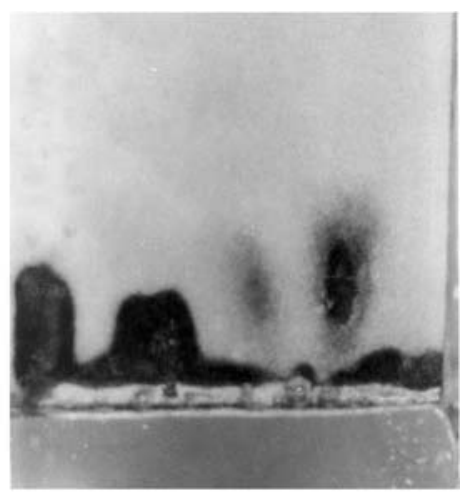

c)

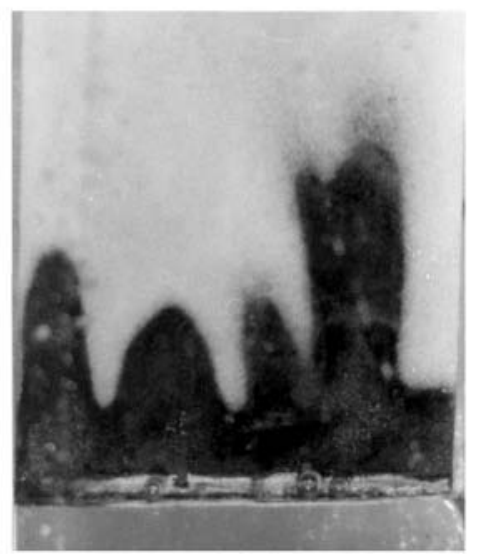

f)

Fig. 3. Morphology of an advancing blackening front in a polycrystalline specimen. Experimental conditions: $T=500^{\circ} \mathrm{C}, U=100 \mathrm{~V}$, (a) $t=20 \mathrm{~min}$, (b) $t=62 \mathrm{~min}$, (c) $t=92 \mathrm{~min}$, (d) $t=112 \mathrm{~min}$, (e) $t=122 \mathrm{~min}$, (f) $t=202 \mathrm{~min}$.

zirconia. The uncharged vacancies may dissociate and thus produce electronic charge carriers which increase the electronic conductivity:

$\mathrm{V}_{\mathrm{O}}^{\times} \rightleftharpoons \mathrm{V}_{\mathrm{O}}^{\cdot}+e^{\prime} \rightleftharpoons \mathrm{V}_{\mathrm{O}}^{\cdot \cdot}+2 e^{\prime}$

Stabilized cubic zirconia contains an appreciable amount of aliovalent impurities (usually $\mathrm{CaO}$ or $\mathrm{Y}_{2} \mathrm{O}_{3}$ ) which introduce a well defined and virtually oxygen activity independent concentration of oxygen vacancies,

$\mathrm{CaO} \rightleftharpoons \mathrm{Ca}_{\mathrm{Zr}}^{\prime \prime}+\mathrm{O}_{\mathrm{O}}^{\times}+\mathrm{V}_{\mathrm{O}}^{\cdot \cdot}$

$\mathrm{Y}_{2} \mathrm{O}_{3} \rightleftharpoons \mathrm{Y}_{\mathrm{Zr}}^{\prime}+3 \mathrm{O}_{\mathrm{O}}^{\times}+\mathrm{V}_{\mathrm{O}}^{*}$

and influence the concentration of electronic charge carriers, see Eq. (2). With Eqs. (1) and (2) and the intrinsic electronic equilibrium

$e^{\prime}+\mathrm{h} \rightleftharpoons 0$

the dependence of the partial conductivities of electrons, electron holes and ions on the oxygen activity can be described as:

$\sigma_{e^{\prime}} \sim a_{\mathrm{O}_{2}}^{-1 / 4}$

$\sigma_{\mathrm{h}} \cdot a_{\mathrm{O}_{2}}^{1 / 4}$

$\sigma_{\text {ion }}=$ const.

Using these relations, a qualitative diagram for the 


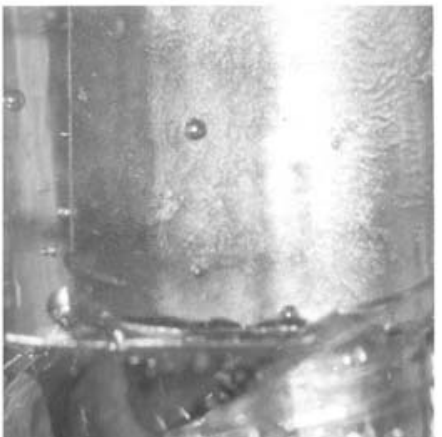

a)

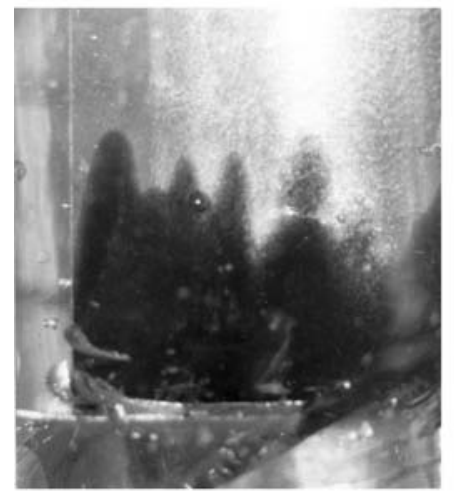

d)

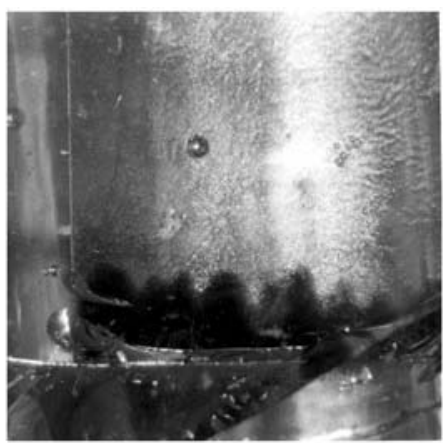

b)

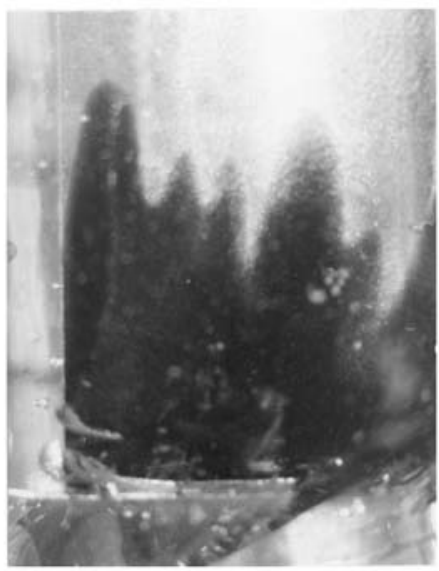

e)

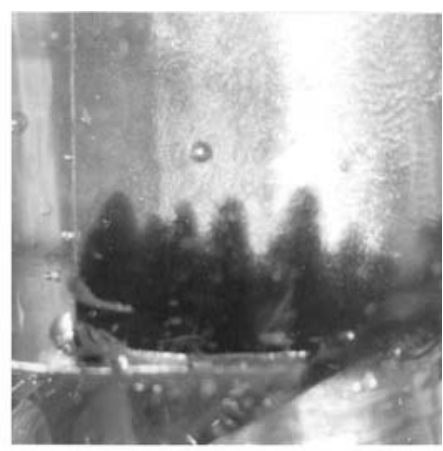

c)

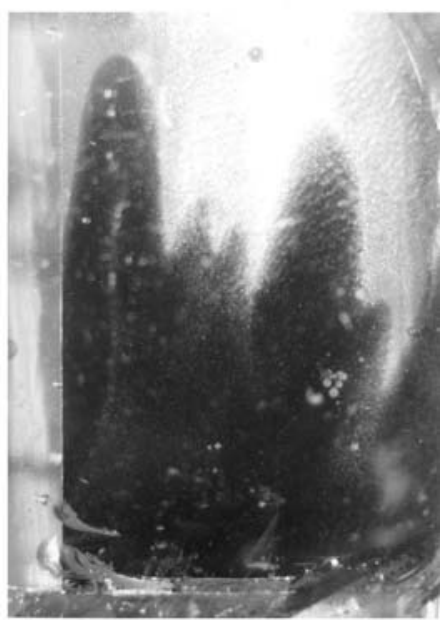

f)

Fig. 4. Morphology of an advancing blackening front in a single crystalline specimen. Experimental conditions: $T=500^{\circ} \mathrm{C}, U=100 \mathrm{~V},(\mathrm{a})$ $t=15 \mathrm{~s}$, (b) $t \approx 2 \mathrm{~min}$, (c) $t \approx 4.5 \mathrm{~min}$, (d) $t \approx 7 \mathrm{~min}$, (e) $t \approx 9.5 \mathrm{~min}$, (f) $t \approx 12 \mathrm{~min}$.

conductivity relations can be constructed. Data for the partial conductivities by Park and Blumenthal [37] are used for the construction of Fig. 5. Due to the virtually oxygen activity independent concentration of oxygen vacancies $\mathrm{V}_{\mathrm{O}}^{. *}$ the ionic conductivity $\sigma_{\text {ion }}$ is approximately constant (Eq. (8)).

The linear flux equation

$j_{i}=-L_{i} \cdot \nabla \tilde{\mu}_{i}=\frac{D_{i} c_{i}}{R T} \cdot \nabla \tilde{\mu}_{i}$

is valid for the different charge carriers $\left(i=e^{\prime}, \mathrm{h}\right.$, $\mathrm{O}^{2-}$ ), with $L_{i}$ representing the phenomenological (diagonal) transport coefficient of charge carrier $i, D_{i}$ denoting its self diffusion coefficient and $c_{i}$ representing the molar concentration of the charge carrier $^{12} \cdot \tilde{\mu}_{i}$ denotes the electrochemical potential of a charge carrier (with $\tilde{\mu}_{i}=\mu_{i}+z_{i} F \varphi ; \mu_{i}$ being the chemical potential; $\varphi$ is the electric potential, $F$ is Faraday's number, and $z_{i}$ is the formal charge number of the charge carrier). $R$ and $T$ denote the gas constant and temperature, respectively.

The cell arrangement is a typical Wagner-Hebb polarization cell with an ion blocking cathode and an anode which is reversible for oxygen exchange. In

\footnotetext{
${ }^{1}$ Independent fluxes of the different charge carriers are assumed, i.e. we set $L_{i j}=0$.

${ }^{2}$ The phenomenological transport coefficient $L_{i}$ of a species $i$ is related to its partial conductivity $\sigma_{i}$ by: $\sigma_{i}=\left(z_{i} F\right)^{2} L_{i}$ 


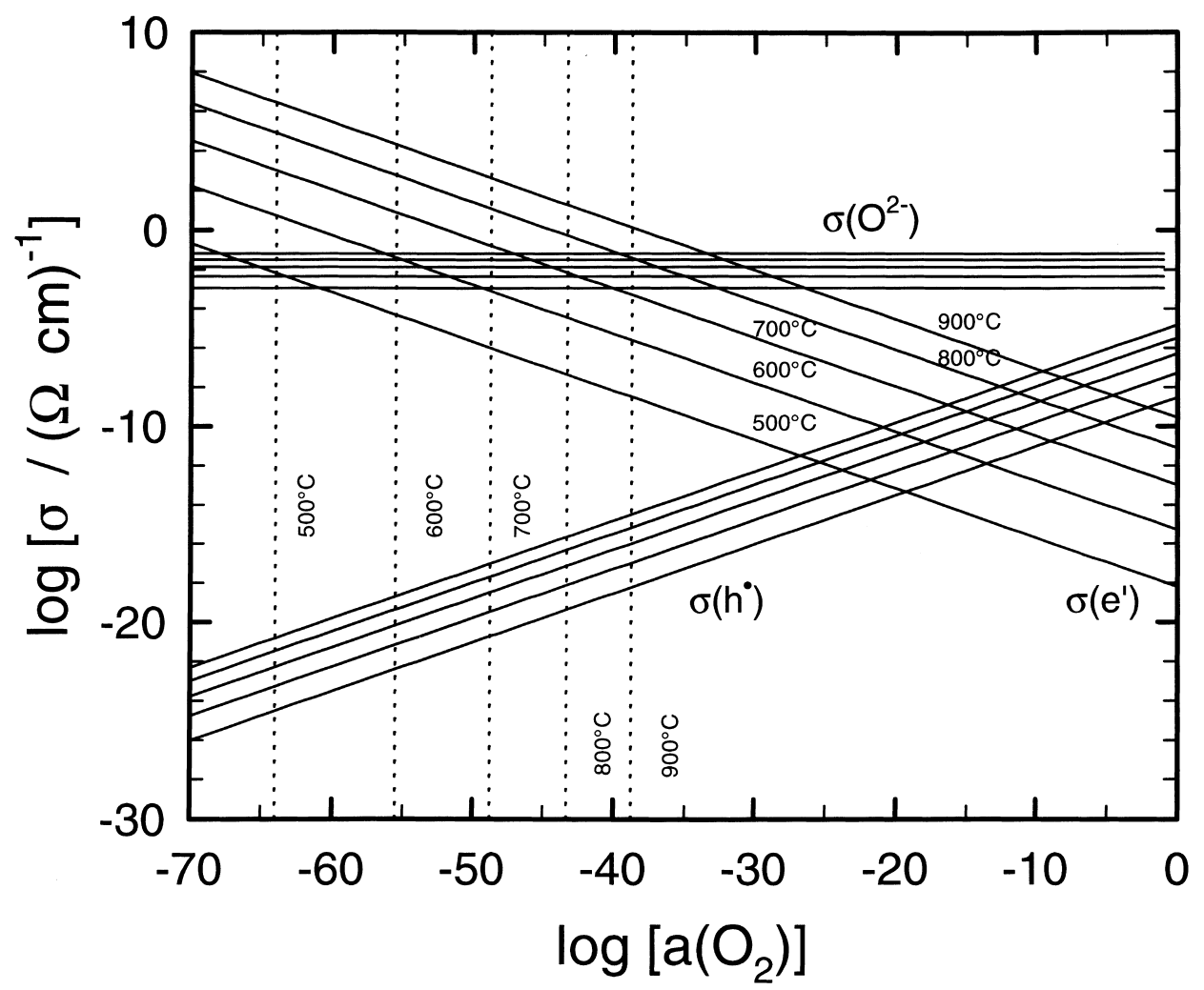

Fig. 5. Conductivity of yttria-stabilized zirconia, according to Ref. [37].

the stationary state, the ion flux vanishes $\left(j_{\mathrm{O}^{2-}}=0\right)$ and from Eq. (9) it follows that

$\nabla \tilde{\mu}_{\mathrm{O}^{2-}}=0$

If it is further assumed that the local concentration $c_{\mathrm{O}^{2-}}$ of oxygen ions is virtually independent from the chemical potential $\mu_{\mathrm{O}_{2}}$ of the component $\mathrm{O}_{2}$ (stabilized zirconia), i.e. $\nabla \mu_{\mathrm{O}^{2-}}=0$, one concludes from Eq. (11) that an ideally polarized crystal without internal reactions is free from an internal (macroscopic) electric field:

$\nabla \varphi=0$

Neglecting possible electronic overvoltages at the electrodes, an applied external voltage $U=\varphi^{b l}(\mathrm{Pt})-$ $\varphi^{r e v}(\mathrm{Pt})$ leads to a difference in the electrochemical potential of electrons,

$U=-\frac{1}{F} \cdot\left[\tilde{\mu}_{e^{\prime}}^{\mathrm{bl}}\left(\mathrm{ZrO}_{2}\right)-\tilde{\mu}_{e^{\prime}}^{\mathrm{rev}}\left(\mathrm{ZrO}_{2}\right)\right]$ in the zirconia crystal between both electrodes, which by use of Eq. (12) is equivalent to

$U=-\frac{1}{F} \cdot\left[\mu_{e^{\prime}}^{\mathrm{bl}}\left(\mathrm{ZrO}_{2}\right)-\mu_{e^{\prime}}^{\mathrm{rev}}\left(\mathrm{ZrO}_{2}\right)\right]$

Assuming local equilibrium within the crystal the local chemical potential of oxygen as a component is introduced

$\mu_{\mathrm{O}_{2}}=2\left(\tilde{\mu}_{\mathrm{O}^{2-}}-2 \tilde{\mu}_{e^{\prime}}\right)$

and one obtains with Eq. (11)

$$
\begin{aligned}
U & =\frac{1}{F} \cdot\left[\mu_{\mathrm{O}_{2}}^{\mathrm{bl}}\left(\mathrm{ZrO}_{2}\right)-\mu_{\mathrm{O}_{2}}^{\mathrm{rev}}\left(\mathrm{ZrO}_{2}\right)\right] \\
& =\frac{R T}{4 F} \cdot \ln \frac{a_{\mathrm{O}_{2}}^{\mathrm{bl}}}{a_{\mathrm{O}_{2}}^{\mathrm{rev}}}
\end{aligned}
$$

Depending on the sign of the applied voltage, the chemical potential of oxygen at the blocking electrode can either be increased or reduced relative to 
the fixed chemical potential at the reversible electrode. Applying a negative voltage, i.e. choosing the blocking electrode as the cathode, the chemical potential of oxygen is reduced at the cathode. As a consequence of Eq. (12) one concludes that electronic transport through the ideally polarized crystal is exclusively driven by the concentration gradient of the electronic charge carriers. Assuming negligible overvoltages at the electrodes, the chemical potential difference across the polarized crystal can be measured directly by $U$.

Different situations are possible, depending on the applied voltage $U$. In Fig. 6a-d the local distribution of the different potentials is depicted schematically for the case of an ideally blocking cathode (case a). Within the crystal the total electric current $i$ must be constant in the stationary state $\left(\nabla \cdot i=-F \nabla \cdot j_{e^{\prime}}=\right.$ $0)$ :

$\nabla \cdot L_{e^{\prime}} \nabla \mu_{e^{\prime}}=0$

Since the electronic transport coefficient $L_{e^{\prime}}$ is a
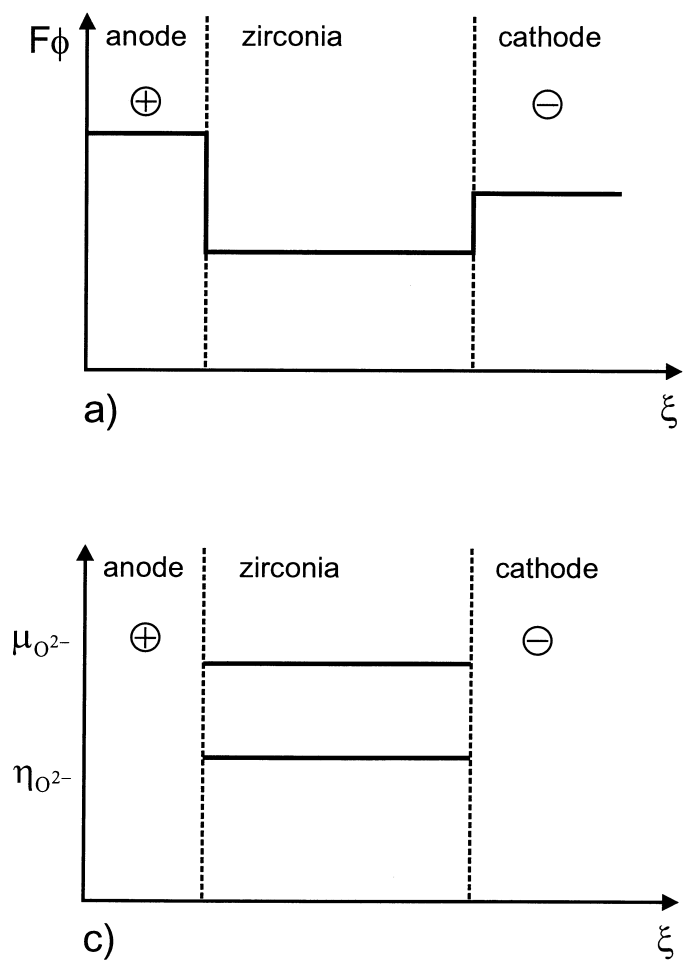

function of the chemical potential $\mu_{e^{\prime}}$ itself $\left(\nabla \mu_{e^{\prime}}=\right.$ $\left.-1 / 4 \nabla \mu_{\mathrm{O}_{2}}\right)$, the profile of the (electro-)chemical potential of electrons is not linear (cf. measurements of potential profiles in $\mathrm{AgBr}$ [38]). The exact shape depends on the relation between the electronic conductivity $\sigma_{e^{\prime}}$ and the chemical potential. The concentration profile of the electronic charge carriers is linear provided that their mobility is concentration independent.

In addition to the complete blocking situation, as characterized by the condition $j_{\mathrm{v}_{\ddot{\mathrm{O}}}}=0, \nabla j_{\mathrm{v}_{\ddot{\mathrm{O}}}}=$ $0, j_{e^{-}} \neq 0$ and $\nabla j_{e^{-}}=0$, two other cases exist which have already been considered by Stilkenböhmer and Schmalzried in the case of $\mathrm{Ag} / \mathrm{AgBr} / \mathrm{Pt}$ cells. In case (b) one assumes that the applied voltage is high enough to cause a cathodic electrode reaction localized at the YSZ/Pt interface. In case (c), the applied voltage is chosen such that the cathodic reaction will advance into the electrolyte bulk rather than being restricted to the interface.

In general, case (b) will correspond to the complete decomposition of the electrolyte at the interface
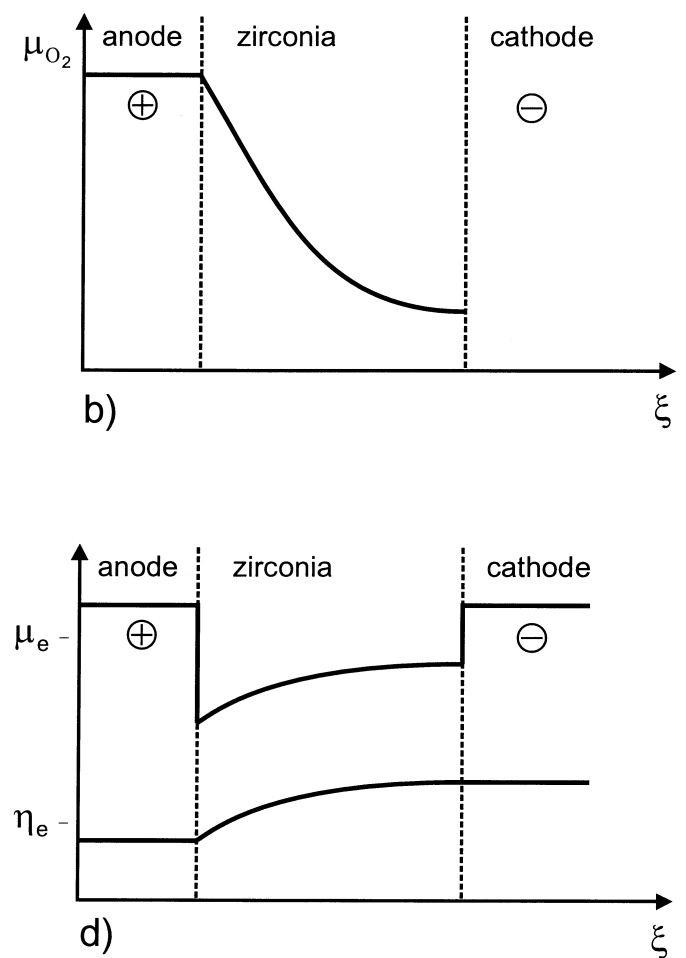

Fig. 6. Local potential variation in the case of an ideally ion blocking cathode without electrochemical reduction of zirconia. 
into metalloid ions which are dragged towards the anode and into the metal component. The deposition of the metal will most probably occur in a whiskeror dendrite-like morphology, in order to short-circuit the cell. So far no direct observation of such electrolysis of anion conductors has been reported to our knowledge. Case (c) corresponds to the injection of charge carriers into the solid electrolyte. An experimental example has been reported by Stilkenböhmer and Schmalzried [35,36]. By polarizing a $\mathrm{AgBr} / \mathrm{Pt}$ anode at low temperatures with voltages higher than $1 \mathrm{kV}$, they observe the formation of bromine filled pores in the solid electrolyte.

Another different situation (case d) is given if a decomposition product can be formed at intermediate potentials within the thermodynamic stability range of the solid electrolyte, i.e. if the electrolyte can be partially reduced due to multivalent metal ions. In the case of YSZ, one might expect the creation of $\mathrm{Zr}$-ions with a valence lower than +4 . At applied voltages which are high enough the electrochemical blackening (reduction) process takes place, starting from the cathode and advancing through the whole crystal towards the cathode. The blackening itself may be caused by different microscopic defects as it is already indicated above.

The most simple reaction scheme assumes the trapping of electrons at oxygen vacancies (see Eq. (2) which may further associate with impurity or zirconium ions $\left(\mathrm{Y}_{\mathrm{Zr}}^{\prime}, \mathrm{Zr}_{\mathrm{Zr}}^{\times}\right)$in order to form more complex point defect clusters. The internal creation of neutral vacancies $\mathrm{V}_{\mathrm{O}}^{\times}$corresponds to the removal of molecular oxygen from the crystal as it is already indicated in Eq. (1). In other terms, the partial reduction of zirconia,

$\mathrm{ZrO}_{2}=\mathrm{ZrO}_{2-\delta}+\frac{\delta}{2} \mathrm{O}_{2}$

can be realized within the crystal by the introduction of neutral combination of vacancies $\mathrm{V}_{\mathrm{O}}^{\cdot}$ and electrons $e^{\prime}$. Since the motion of oxygen vacancies $\mathrm{V}_{\mathrm{O}}^{*}$ is coupled to the counterwise motion of oxygen ions $\mathrm{O}_{\mathrm{O}}^{\times}$, these will move from the reduction site to the surface of the crystal to form molecular oxygen. A simplified reaction scheme which is based on the above considerations is depicted in Fig. 7.

Another reaction scheme is based on the trapping of electrons at zirconium ions $\mathrm{Zr}_{\mathrm{Zr}}^{\times}$forming ions with an absolute charge number lower than +4 (e.g. formation of trivalent ions, $\mathrm{Zr}_{\mathrm{Zr}}^{\prime}$ ). Stressing this particular reaction one could even formulate the complete reduction of zirconium ions to the neutral metal state ( $\mathrm{Zr}_{\mathrm{Zr}}^{\prime \prime}$ ') ). By further association of two oxygen vacancies this could lead to the formation of a zirconium metal atom within a microscopic pore inside the crystal:

$\mathrm{Zr}_{\mathrm{Zr}}^{\prime \prime \prime}+2 \mathrm{~V}_{\mathrm{O}}^{. *} \rightleftharpoons\left\{\mathrm{Zr}_{\mathrm{Zr}}^{\prime \prime \prime}+2 \mathrm{~V}_{\mathrm{O}}^{\cdot \cdot}\right\}^{\times} \equiv \mathrm{Zr}$

For the precipitation of metal clusters, as is discussed frequently, at least a small number of these metal atoms would have to aggregate. Due to the very slow mobility of cations within the fluorite-type structure this appears unlikely, in particular at relatively low temperatures, and to date no clear evidence is given for such process.

Metal impurities like $\mathrm{Ti}_{\mathrm{Zr}}^{\times}$may act also as traps for electronic defects and cause color changes with decreasing oxygen activity. However, the microscopic nature of the reduction product is of no meaning for the phenomenological analysis of the reaction. Rather it is sufficient to formulate a reaction which consumes electrons $e^{\prime}$ and oxygen vacancies $\mathrm{V}_{\mathrm{O}}^{\cdot}$, producing oxygen ions $\mathrm{O}_{\mathrm{O}}^{\times}$which leave the reaction zone:

$\mathrm{ZrO}_{2}+2 \delta e^{\prime}+\delta \mathrm{V}_{\mathrm{O}}^{\cdot *}=\mathrm{ZrO}_{2-\delta}+\delta \mathrm{O}_{\mathrm{O}}^{\times}$

Finally, the stability of the advancing reduction front has to be discussed. From the condition of a constant total electric current $(\nabla \cdot i=0)$, one obtains the following relation between the ionic and electronic fluxes in the reaction zone: ${ }^{3}$

$j_{e^{\prime}}^{\prime \prime}-j_{e^{\prime}}^{\prime}=2\left(j_{\mathrm{v}_{0}}^{\prime \prime}-j_{\mathrm{v}_{0}}^{\prime}\right)=-2\left(j_{\mathrm{O}_{\mathrm{O}}^{\times}}^{\prime \prime}-j_{\mathrm{O}_{\mathrm{O}}^{\times}}^{\prime}\right)$

We can safely assume that the electronic conductivity $\sigma_{e^{\prime}}^{\prime}$ in the unreduced zirconia is several orders of magnitude lower than the ionic conductivity $\sigma_{\mathrm{v}_{\mathrm{o}}}^{\prime}$. Thus, the charge transport in the unreduced material is only due to oxygen vacancies $\left(t_{\mathrm{v}_{\ddot{\mathrm{O}}}}^{\prime}=\sigma_{\mathrm{v}_{\ddot{\mathrm{O}}}}^{\prime} /\left(\sigma_{\mathrm{v}_{\ddot{\mathrm{O}}}}^{\prime}+\sigma_{e^{\prime}}^{\prime}\right) \approx 1\right)$ [37]. Vice versa, we

${ }^{3}$ In the following, all quantities which are related to the unreduced region are marked with a prime and all quantities which are related to the reduced (blackened) region of zirconia are marked with a double prime. 

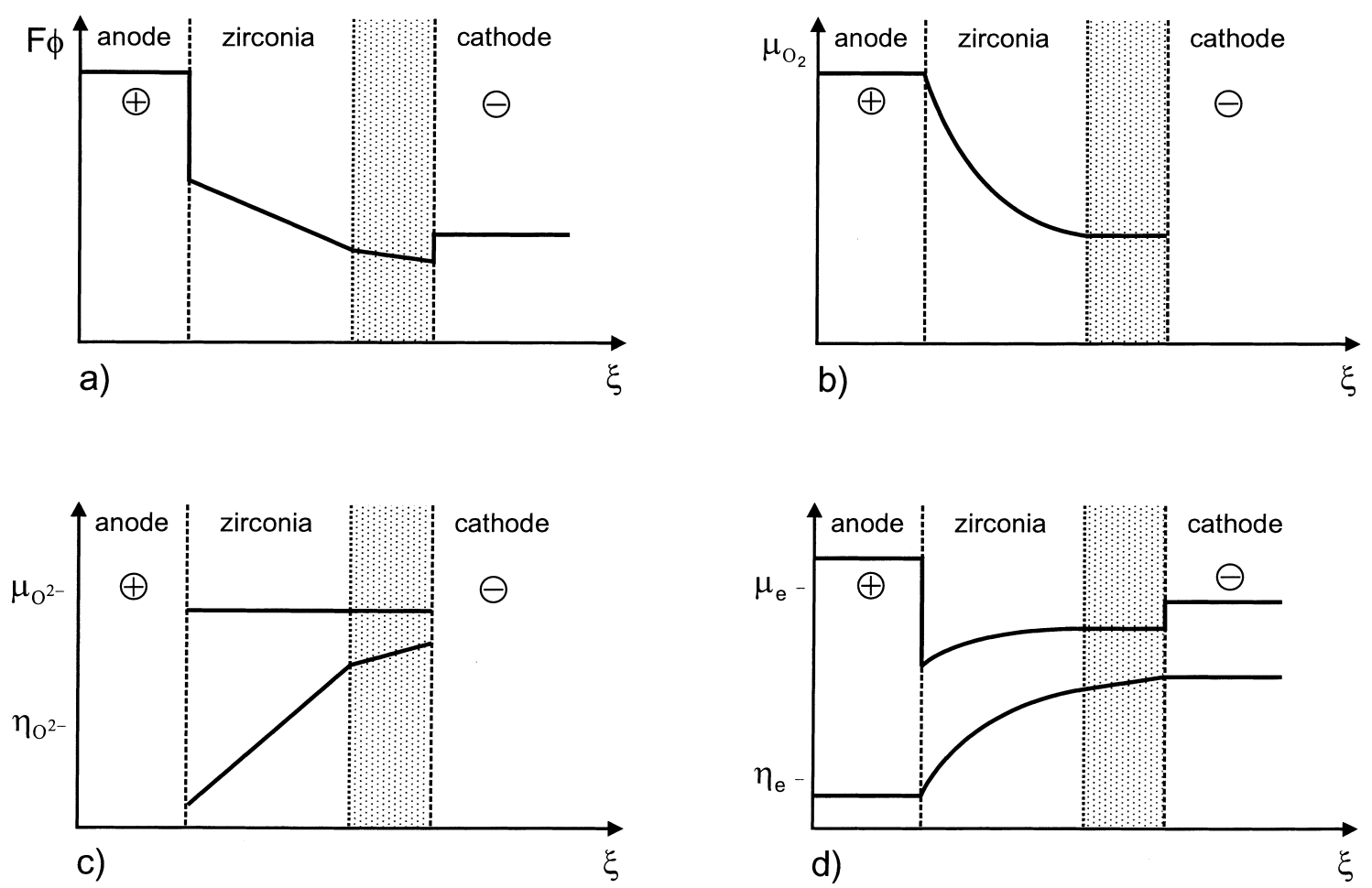

Fig. 7. Reaction scheme for the electrochemical reduction of zirconia.

assume that in the reduced (blackened) zirconia the electronic conductivity exceeds the ionic conductivity $\left(t_{e^{\prime}}^{\prime \prime}=1-t_{\mathrm{v}_{0} \cdot}^{\prime \prime} \approx 1\right)$. Hence, Eq. (21) can be simplified as:

$j_{e^{\prime}}^{\prime \prime} \approx-2 j_{\mathrm{v}_{\mathrm{o}}}^{\prime}$

Taking Eq. (20) and Eq. (22) into account one obtains the following expression for the velocity of the moving boundary between reduced and unreduced zirconia $\left(c_{e^{\prime}}^{\prime \prime}-c_{e^{\prime}}^{\prime} \approx 2 \delta / V_{m}\right.$ and $c_{\mathrm{v}_{0}^{\prime}}^{\prime}-c_{\mathrm{v}_{0}^{\prime}}^{\prime \prime} \approx \delta /$ $V_{m}$ ) [32]:

$v_{b} \approx \frac{j_{e^{\prime}}^{\prime \prime}}{2 \delta / V_{m}} \approx-\frac{j_{\mathrm{V}_{\mathrm{o}}}^{\prime}}{\delta / V_{m}}$

Once reduction of the material occurs, an ionic flux in the unreduced zone is necessarily observed, and Eq. (12) is not strictly valid anymore. If one assumes that the chemical potential of vacancies in the unreduced phase and of electrons in the reduced phase is nevertheless nearly constant $\left(\nabla \mu_{e}^{\prime \prime}, \approx\right.$
$0, \nabla \mu_{\mathrm{v}_{\mathrm{o}}}^{\prime} \approx 0$ ), both fluxes (Eq. (9)) depend only on the gradient $\nabla \varphi$ of the electric potential:

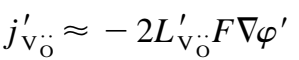

$j_{e^{\prime}}^{\prime \prime} \approx-L_{e^{\prime}}^{\prime \prime} F \nabla \varphi^{\prime \prime}$

Applying Eq. (22) one derives the following (trivial) relation between the electrical potential gradients in both phases:

$\frac{\nabla \varphi^{\prime}}{\nabla \varphi^{\prime \prime}}=\frac{L_{e^{\prime}}^{\prime \prime}}{4 L_{\mathrm{V}_{\ddot{\mathrm{O}}}^{\prime}}^{\prime}}=\frac{\sigma_{e^{\prime}}^{\prime \prime}}{\sigma_{\mathrm{V}_{\mathrm{O}}^{\prime}}^{\prime}}$

In Fig. 8a-d the local distributions of the different potentials in case of electrochemical reduction are depicted schematically.

With respect to the morphological development of the reduction front two cases can be distinguished: (a) the blackening requires an oxygen activity at which the electronic conductivity $\sigma_{e^{\prime}}^{\prime \prime}$ of zirconia is already higher than the ionic conductivity $\sigma_{\mathrm{V}_{0}}^{\prime}$. In this case the voltage drop $\Delta \varphi^{\prime \prime}$ across the blackened 


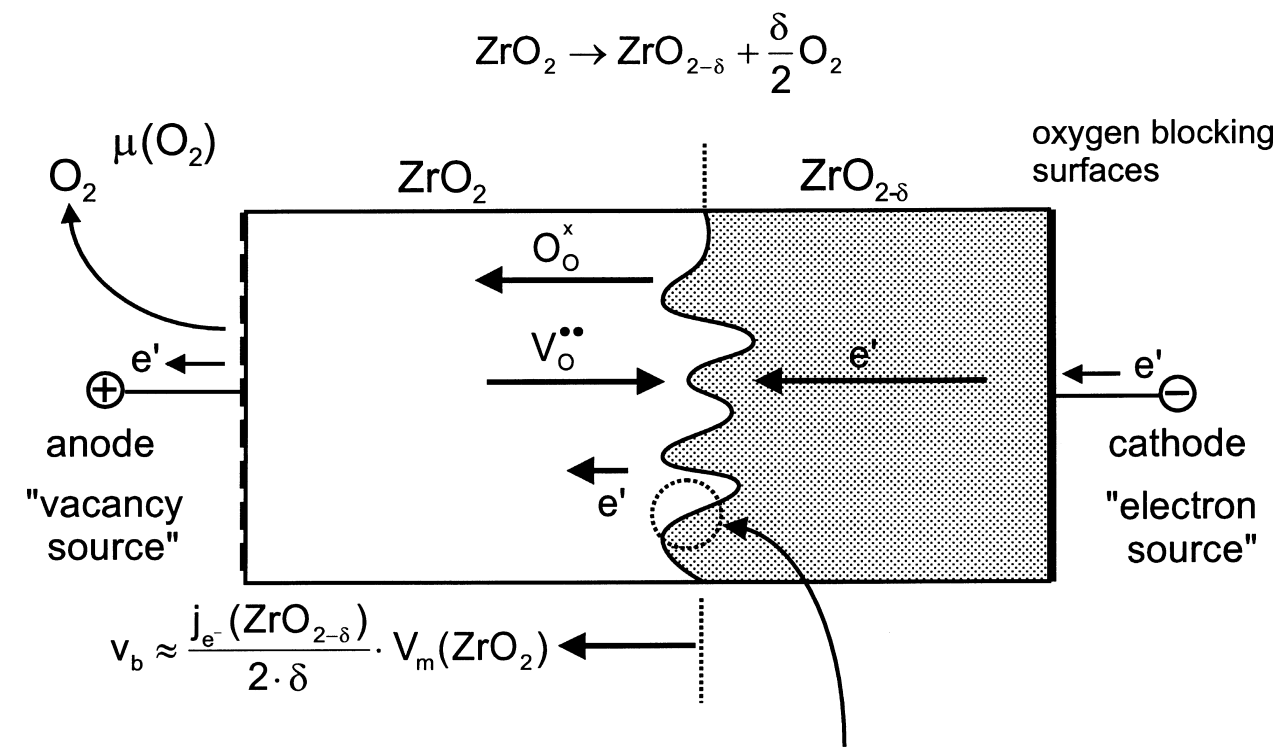

oxidation:

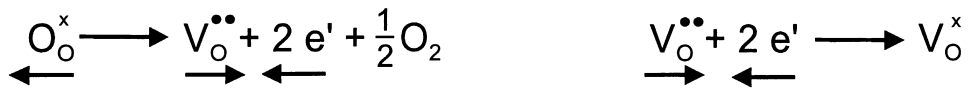

$$
\begin{aligned}
& 2 \mathrm{~V}_{\mathrm{O}}^{\mathrm{x}}+\mathrm{Zr}_{\mathrm{Zr}}^{\mathrm{x}} \longrightarrow \mathrm{Zr}(\mathrm{Me}) \\
& \stackrel{\mathrm{V}_{\mathrm{O}}^{\bullet \bullet}}{\longrightarrow}+\mathrm{e}^{\prime}+\mathrm{Zr}_{\mathrm{Zr}}^{\mathrm{x}} \longrightarrow\left\{\mathrm{V}_{\mathrm{O}}^{\bullet \bullet} \mathrm{Zr}_{\mathrm{Zr}}^{\prime}\right\}^{\bullet}
\end{aligned}
$$

Fig. 8. Local potential variation in the case of an ideally ion blocking cathode with electrochemical reduction of zirconia.

zirconia is smaller than the voltage drop $\Delta \varphi^{\prime}$ across the unreduced zirconia. Thus, the flux $j_{\mathrm{V}_{0}}^{\prime}$ of ions and the related electric field $E^{\prime}=-\nabla \varphi^{\prime}$ in the unreduced zirconia controls the rate of the reduction process. The corresponding potential distribution is depicted schematically in Fig. 8a. Once a small disturbance of an originally planar reaction front occurs in the direction of the moving reduction front, the local electric field $E^{\prime}=-\nabla \varphi^{\prime}$ increases at this disturbance, see Fig. 9. Thus, the vacancy flux $j_{\mathrm{v}_{0}^{\prime}}^{\prime}$ which controls the advancement of the reduction front increases locally and the disturbance grows.

(b) The blackening occurs at a chemical potential at which the ionic conductivity $\sigma_{\mathrm{V}_{0}}^{\prime}$ in the unreduced zirconia is higher than the electronic conductivity $\sigma_{e^{\prime}}^{\prime \prime}$

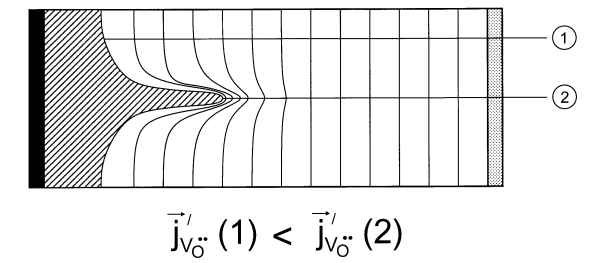

(1)

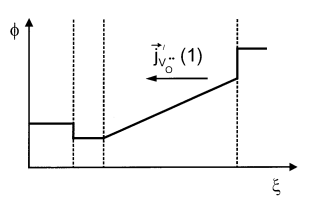

(2)

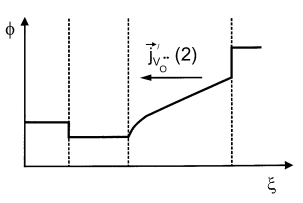

Fig. 9. Variation of the electric field induced by a disturbance of the planar geometry of the advancing reduction front. 
in the reduced zirconia. In this case the flux $j_{e^{\prime}}^{\prime \prime}$ of electrons through the reduced zone is rate determining for the advancement of the reduction front. A similar argument as in case (a) leads to the conclusion that the reduction front remains stable in this case.

Qualitative rules for the discussion of the morphological stability of a moving boundary are given in Ref. [32]. A so-called 'reactive flux' is defined which controls the advancement of the boundary and which consumes most of the external driving force, see Fig. 10.

In case (a) the flux $j_{\mathrm{V}_{0}}^{\prime}$ of oxygen vacancies in the unreduced zone can be identified as the reactive flux. The velocity vector $v_{b}$ of the boundary is directed toward the unreduced zone in which the reactive flux $j_{\mathrm{V}_{\ddot{\mathrm{O}}}}^{\prime}$ occurs, and the boundary moves in an unstable manner. In case (b) the flux $j_{e^{\prime}}^{\prime \prime}$ of electrons in the reduced zone can be identified as the reactive flux. The velocity vector $v_{b}$ of the boundary is directed toward the phase which does not contain the reactive flux, and the boundary remains stable.

Given that the reduction front becomes always morphologically unstable, we conclude that the electronic conductivity $\sigma_{e^{\prime}}^{\prime \prime}$ in the reduced zirconia is definitely higher than the ionic conductivity $\sigma_{\mathrm{v}_{\mathrm{o}}}^{\prime}$ in the unreduced material. This may also be inferred from Fig. 5 which shows data for the partial conductivities in yttria-stabilized zirconia (taken from [37]) together with calculated data for the oxygen activity at the equilibrium $\mathrm{Zr} / \mathrm{ZrO}_{2}$. However, the low

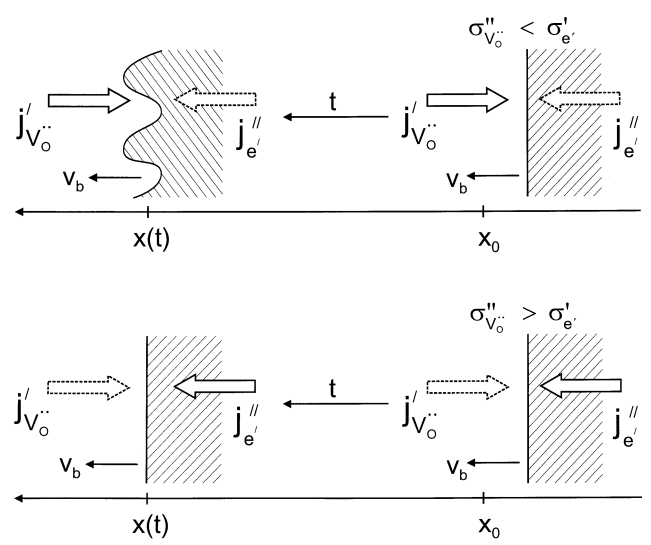

Fig. 10. Stability criteria for moving boundaries according to Ref. [32]. activity data in Fig. 5 are only extrapolated from measurements at higher activities, and the possible influence of the yttrium content on the equilibrium $\mathrm{Zr} / \mathrm{ZrO}_{2}$ has been neglected. Thus, Fig. 5 has to be regarded with care.

As a consequence of the increased electronic conductivity in reduced zirconia, the transport coefficient $L_{\mathrm{O}}$ for the oxygen component will also be large, due to both the high ionic and electronic conductivity $\sigma_{\mathrm{v}_{\ddot{\mathrm{o}}}}^{\prime \prime}$ and $\sigma_{e^{\prime}}^{\prime \prime}\left(\sigma_{\mathrm{v}_{\ddot{\mathrm{o}}}}^{\prime \prime} \approx \sigma_{\mathrm{v}_{\ddot{\mathrm{o}}}}^{\prime}\right)$. Together with a high thermodynamic factor this leads to a high chemical diffusion coefficient $\tilde{D}_{\mathrm{O}}$ which causes the observed homogeneously blackened product region.

\section{Discussion}

According to Eq. (16), the EMF of a partially reduced zirconia specimen offers information on the oxygen activity within the reduced material. The observed maximum (open cell) EMF of approximately $2.4 \mathrm{~V}$ at $500^{\circ} \mathrm{C}$ corresponds to an oxygen activity of $\log a_{\mathrm{O}_{2}} \simeq-62$. From thermodynamic data [39] we estimate an oxygen activity $\log a_{\mathrm{O}_{2}} \simeq-64$ for the equilibrium between pure zirconium metal and pure zirconia. Thus, the metal activity within the blackened zone during the reduction is very close to the activity of zirconium metal. However, under open cell conditions, the initially high EMF decreases quickly indicating back diffusion of the oxygen component through the unreduced material. Nevertheless, it could not be observed that the blackened zone becomes lighter or smaller when the EMF decreases under open cell conditions.

The low oxygen activity which is obtained within the blackened material proves the oxygen-blocking function of the glass cover. Using glass-encapsulation it is thus possible to prepare reduced zirconia with a well defined and very low oxygen activity. However, the study of the reduced material is difficult. The application of local probes within the encapsulated polarisation cell would provide information on the electronic conductivity but failed so far due to the experimental difficulties. Locally resolved optical transmission spectroscopy (in situ) could provide further experimental information.

An early study on the potential distribution in electrochemically polarized zirconia has been re- 
ported by Casselton et al. [1,2]. A number of thin platinum wires were attached along a zirconia specimen in order to detect the local electric field during electroreduction. However, the value of these measurements is restricted since the surfaces of the specimen were not closed for the exchange of oxygen. Thus, the reported profiles provide no reproducible information on the potential distribution under ideal ion blocking conditions.

Waser et al. studied the field distribution during electrochemical degradation of $\mathrm{SrTiO}_{3}$ by means of electron beam deflection in an SEM and by electrooptic measurements. Detailed information on the reduction kinetics and the rate-determining steps could thus be obtained. As in the present case, the migration of oxygen vacancies limits the reduction rate. However, due to a low intrinsic concentration level of vacancies, the electrochemical polarisation leads to serious gradients in the vacancy concentration. In the case of YSZ or CSZ the high concentration of oxygen vacancies caused by doping prevents the establishment of a serious vacancy concentration gradient.

As indicated above, the increased electronic conductivity in the reduced material results in a high chemical diffusion coefficient. To estimate the chemical diffusion coefficient in strongly reduced zirconia we assume that the ionic conductivity of the reduced material does not differ significantly from the conductivity of the unreduced material. As conductivity measurements by Levy et al. [20] show this is not strictly true, i.e. the production of additional vacancies and electronic charge carriers influences the ionic conductivity. The reason for this interaction is yet not clarified but the increase of the dielectric number by the introduction of electronic charge carriers might be an explanation. The change in the dielectric number would cause an increasing dissociation of associates between impurity ions and oxygen vacancies, thus increasing the concentration of mobile oxygen vacancies. We further assume that the electronic conductivity is considerably higher than the ionic conductivity of the reduced material, and that in contrast to the unreduced zirconia the ionic flux controls the flux of the oxygen component. A difficult estimate concerns the thermodynamic factor of reduced zirconia. No quantitative information on the deviation from stoichiometry in strongly reduced zirconia is available. Only two experimental studies report on the deviation from nonstoichiometry in reduced zirconia. Xue and Dieckmann [40] report data on the deviation from stoichiometry in pure and in commercial zirconia at high temperatures $\left(900^{\circ} \mathrm{C}<T<1400^{\circ} \mathrm{C}\right)$ in the oxygen activity range $-13<\log a_{\mathrm{O}_{2}}<0$. The observed deviations from stoichiometry are in the order of $10^{-5}$ even at these high temperatures. Levy et al. [20] measured the degree of nonstoichiometry in yttria-stabilized zirconia and obtained values in the range between $10^{-3}$ and $10^{-2}$ at temperatures between 500 and $1000 \mathrm{~K}$, using different reducing gas mixtures but without denoting the corresponding oxygen activities. From the general equation

$$
\begin{aligned}
& \tilde{D}_{\mathrm{O}}=\frac{L_{\mathrm{O}^{2-}} \cdot L_{e^{\prime}}}{4 L_{\mathrm{O}^{2-}}+L_{e^{\prime}}} \cdot \frac{\partial \mu_{\mathrm{O}}}{\partial c_{\mathrm{O}}} \\
& =\frac{1}{4 F^{2}} \cdot \frac{\sigma_{\mathrm{O}^{2-}} \cdot \sigma_{e^{\prime}}}{\sigma_{\mathrm{O}^{2-}}+\sigma_{e^{\prime}}} \cdot \frac{R T}{c_{\mathrm{O}}} \cdot \frac{\partial \ln a_{\mathrm{O}}}{\partial \ln c_{\mathrm{O}}}
\end{aligned}
$$

for the chemical diffusion coefficient (neglecting interactions between electrons and oxygen vacancies) we obtain the relation

$\tilde{D}_{\mathrm{O}} \approx-\sigma_{\mathrm{O}^{2-}} \cdot \frac{V_{m}}{8 F^{2}} \cdot \frac{R T}{\delta} \cdot \frac{\partial \ln a_{\mathrm{O}_{2}}}{\partial \ln \delta}$

by applying the above given assumptions. Assuming a value of $\delta \simeq 10^{-3}$ for the metal deficit and a value of $\partial \ln \delta / \partial \ln a_{\mathrm{O}_{2}} \simeq-1 / 4$, and using values of $V_{m} \approx 20$ $\mathrm{cm}^{3} / \mathrm{mol}$ and $\sigma_{\mathrm{O}^{2-}}=1.15 \times 10^{-3}(\Omega \mathrm{cm})^{-1}$, we estimate the chemical diffusion coefficient as $\tilde{D}_{\mathrm{O}} \approx 8 \times$ $10^{-6} \mathrm{~cm}^{2} / \mathrm{s}$ at $T=500^{\circ} \mathrm{C}$. Obviously, our estimation depends critically on $\delta$ and on the ionic conductivity. It should therefore be regarded only as a rough estimate.

The observed morphological instability of the reaction front is directly related to the transport properties of zirconia under different thermodynamic conditions. The conductivity diagram (see Fig. 5) indicates that the change from predominant ionic conductivity to predominant electronic conductivity generally occurs at an oxygen activity below the equilibrium activity $a_{\mathrm{O}_{2}}(\mathrm{Zr} / \mathrm{YSZ})$. Thus, at least in the depicted temperature region the reduction should always proceed in a morphologically unstable way.

No reliable information on the relation between 
the blackening intensity and the oxygen activity is available. Thus, it is not possible to relate the observed blackening front with a definite oxygen activity. However, assuming a high chemical diffusion coefficient within the reduced material one has to conclude that the blackened region is virtually homogeneous in respect to the oxygen activity. A relatively sharp boundary between reduced and unreduced material results. As already proposed by Levy et al. [20], the advancing and homogeneous blackened region can be seen as an 'internal' electrode.

The different reduction rates for single and polycrystalline material highlights the role of grain boundaries as kinetic barriers for oxygen ion conduction. Since the velocity of the reduction front depends critically on the ionic flux in the unreduced material, the slower reduction of polycrystalline material indicates high grain boundary resistances. It has to be mentioned that the grain boundary resistance is of major importance for the case of electrochemical resistance degradation in titanate ceramics. Fine grained ceramics exhibit much slower degradation rates than coarse grained ceramics or single crystals. This has been demonstrated both experimentally and numerically by Waser et al. [34].

\section{Conclusions}

The morphological development of moving reaction fronts is of practical interest in the field of solid state reactions. The investigated reduction process can be regarded as an example for electrochemically induced internal reactions [32]. The morphological instability can be understood on the basis of simple kinetic arguments.

Under well defined boundary conditions the dependence of the morphology of the reaction front on experimental parameters has been studied. The occurrence of the morphological instability itself bears information on the transport properties of strongly reduced zirconia. Thus, blackened zirconia exhibits a higher electronic conductivity than the total conductivity of unreduced material. A direct consequence is a high transport coefficient for oxygen which causes an almost uniform chemical potential within the blackened material. Grain boundaries can be regarded as barriers for oxygen ion transport, and directly related to this fact, ceramic specimens show much slower blackening rates than single crystalline specimens under identical conditions.

\section{Acknowledgements}

Helpful assistance in the selection of suitable glass material by Dr. Pfeiffer (Schott Glaswerke, Mainz) is gratefully acknowledged. We thank Prof. H. Schmalzried for valuable comments and suggestions.

\section{References}

[1] R.E.W. Casselton, Electronic MHD 4 (1968) 2951.

[2] R.E.W. Casselton, M.D.S. Watson, Sci. Ceram. 4 (1968) 349.

[3] D.A. Wright, J.S. Thorp, A. Aypar, H.P. Buckley, J. Mater. Sci. 8 (1973) 876.

[4] D. Nagle, V.R. PaiVerneker, A.N. Petelin, G. Groff, Mater. Res. Bull. 24 (1989) 619.

[5] V.R. PaiVerneker, A.N. Petelin, F.J. Crowne, D.C. Nagle, Phys. Rev. B 40(12) (1989) 8555.

[6] C. Bonola, P. Camagni, P. Chiodelle, G. Samoggia, Rad. Eff. Def. Solids 119-121 (1991) 457.

[7] R.E.W. Casselton, J.S. Thorp, D.A. Wright, Proc. Br. Ceram. Soc. 19 (1971) 265.

[8] J.S. Thorp, A. Aypar, J.S. Ross, J. Mater. Sci. 7 (1972) 729.

[9] J. Shinar, D.S. Tannhauser, B.L. Silver, Solid State Commun. 56(2) (1985) 221.

[10] J. Genossar, D.S. Tannhauser, Cryst. Latt. Def. Amorph. Mater. 16 (1987) 1.

[11] J. Genossar, D.S. Tannhauser, Solid State Ionics 28-30 (1988) 503.

[12] J.S. Moya, R. Moreno, J. Requena, J. Am. Ceram. Soc. 71(11) (1988) C479.

[13] C.B. Azzoni, A. Paleari, Phys. Rev. B 40(10) (1989) 6518.

[14] C.B. Azzoni, A. Paleari, Phys. Rev. B 40(13) (1989) 9333.

[15] R.W. Rice, J. Am. Ceram. Soc. 74(7) (1991) 1745.

[16] J. Soria, J.S. Moya, J. Am. Ceram. Soc. 74(7) (1991) 1747.

[17] R. Ben-Michael, D.S. Tannhauser, J. Genossar, Phys. Rev. B 43(10) (1991) 7395.

[18] V.M. Orera, R.I. Merino, Y. Chen, R. Cases, P.J. Alonso, Rad. Eff. Def. Solids 119-121 (1991) 907.

[19] R.E.W. Casselton, J. Appl. Electrochem. 4 (1974) 25.

[20] M. Levy, J. Fouletier, M. Kleitz, J. Electrochem. Soc. 135(6) (1988) 1584.

[21] F.K. Moghadam, T. Yamashita, D.A. Stevenson, J. Mater. Sci. 18 (1983) 2255.

[22] J.S. Thorp, H.P. Buckley, J. Mater. Sci. 8 (1973) 1401.

[23] R.E.W. Casselton, J. Penny, M.J. Reynolds, Trans. J. Br. Ceram. Soc. 76(3) (1971) 115. 
[24] J.M. Farley, J.S. Thorp, J.S. Ross, G.A. Saunders, J. Mater. Sci. 7 (1972) 475.

[25] S. Yoshioka, T. Miyazaki, Bull. Jpn. Soc. Prec. Eng. 24(4) (1990) 258.

[26] T.E. Warner, R. Janes, P.P. Edwards, J. Mater. Sci. Lett. 10 (1991) 937.

[27] K.-D. Becker, Ber. Bunsenges. Phys. Chem. 99 (1995) 658

[28] C. Deportes et al., Electrochimie des Solides, Presses Universitaire des Grenoble, Grenoble, France.

[29] T. Große, H. Schmalzried, Z. Phys. Chem. N.F. 172 (1992) 197.

[30] M. Hebb, J. Phys. Chem. 20 (1952) 185.

[31] C. Wagner, Proc. Int. Committee Electrochem. Thermodyn. Kinetics (C.I.T.C.E.) 7 (1955) 361.

[32] H. Schmalzried, Chemical Kinetics of Solids, Verlag Chemie, Weinheim, 1995.
[33] H. Schmalzried, M. Backhaus-Ricoult, Prog. Solid State Chem. 22 (1993) 1.

[34] R. Waser, T. Bajatu, K.-H. Härdtl, J. Am. Ceram. Soc. 73 (1990) 1645, 1654, 1663.

[35] U. Stilkenböhmer, Ph.D. Thesis, University of Hannover, 1994.

[36] U. Stilkenböhmer, H. Schmalzried, Phys. Stat. Sol. (a) 146 (1994) 31.

[37] J.-H. Park, R.N. Blumenthal, J. Electrochem. Soc. 136(10) (1989) 2867.

[38] C. Rosenkranz, J. Janek, Solid State Ionics 82 (1995) 95.

[39] O. Kubaschewski, C.B. Alcock, P.J. Spencer, Materials Thermochemistry, 6th ed., Pergamon Press, Oxford, 1993.

[40] J. Xue, R. Dieckmann, Adv. Ceram. 24 (1993) 106. 